\title{
Effect of ethanol in mouthwashes On the surface hardness of a dental resin composite material
}

\author{
Decky J. Indrani, Triaminingsih S, Nurvanita, Andhesti, Noviana
}

Department of Dental Materials Science Faculty of Dentistry Universitas Indonesia

\begin{abstract}
Previous study has shown that dental resins exhibited changes in the surface hardness when soaked in alcoholic beverages. As ethanol was one of the components contained in some commercial mouthwashes, therefore, the present study aimed at the effect of ethanol in some mouthwashes on the surface hardness of a dental resin composite. Specimens were prepared from a light cured dental resin composite. The specimens were then immersed in some commerial mouthwashes for periods up to 14 days. The surface hardness of the specimens were then measured using a Microhardness tester. The results displayed reductions in surface hardness $(p<0,01)$, upon one day of immersing the specimens in Bactidol $\circledast$, Listerine ${ }^{\circ}$ or Minosepe $₫$ mouthwash, respectively. The surface hardness curves from each mouthwash demonstrated a similar pattern which approached a plateau near 2 weeks. It can be concluded that the ethanol-containing mouthwashes appeared to have more implication on surface hardness of the dental resin composite and the effect of immersing in the mouthwashes was time-dependent; it decreased sharply on the first day of immersion, however, further changes, did not exist.
\end{abstract}

Key words: dental resin composite, mouthwash, surface hardness

\section{INTRODUCTION}

Dental resin composites have been widely developed and successfully used in mouth as restoratives for decades. In mouth, resin composite materials have always been in contact with saliva. Due to the polarity of polymer materials that cause the susceptibility of resin composite materials to water sorption, most resin composite materials absorbed water. ${ }^{1-4}$ Water molecules in the form of $\mathrm{H}^{+}$or $\mathrm{OH}^{-}$ions from the surrounding environment, i.e. saliva in mouth, may diffuse into the matrix or the resin composite material. Located within the resin matrix, water molecules enable the atom in the resin structure to be rendered more mobile. This can actually maintain a reasonable stability of resin structure. ${ }^{2}$ Water exposure into the resin molecules structure in a long-term survival, however, may lead to negative effects. The mechanism behind the degradation process can be explained by a self-catalytic reaction as was explained by Soderholm. ${ }^{5}$

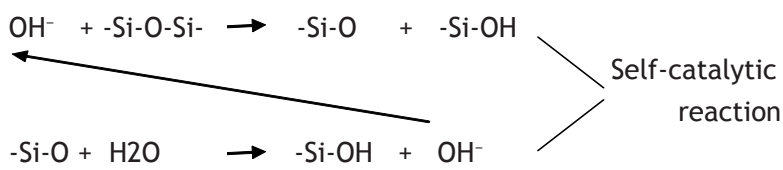

Accordingly, $\mathrm{OH}^{-}$ions with a silica surface break siloxane bonds to form silanol groups as shown through a figure below: 


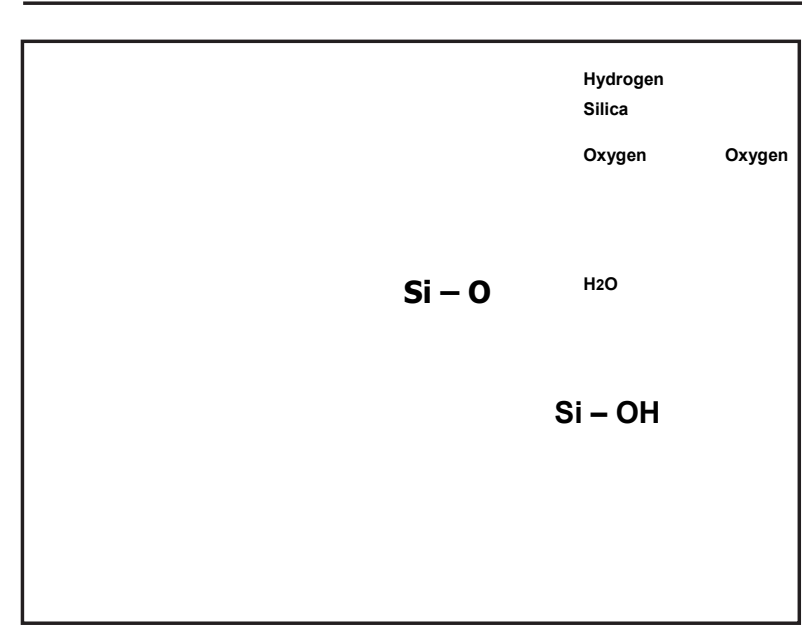

Figure 1. Breakage of Si-O-Si bridge.

At a sufficient concentration, $\mathrm{OH}^{-}$ions interact with the siloxane network, and the reaction becomes autocatalytic.

Water and beverages containing alcohol has been used as solvents to simulate accelerated ageing of resin composite materials. Seventyfive persent of ethanol/water solution has been the closest match in solubility parameter results in softening of the resin matrix..$^{6,7,8}$ The authors assumed that the decrease in the surface hardness was due to a chemical softening of the resin matrix by water molecules. Ethanol ${ }^{5,9}$ and also alcoholic beverages $^{10}$ have been demonstrated to lower the fracture toughness and further increase the wear, respectively. Ethanol in wine was proposed to swell the resin matrix that changed the mechanical values. ${ }^{9,11}$ As alcohol may also elicited from mouthwashes, research has been established whether this environment has influence to destruct the resin matrix of restorative composites. In the previous work significant differences occurred among the surface hardness values obtained from resin composite specimens immersed in Listerine mouthwash simulating gargling the mouthwash for 2 minutes twice a day up to 14 days when compared to those immersed in distilled water. While the previous work experimented a relative short-term time exposure in Listerine, the present study simulated a longer time exposure and in three commercial mouthwashes. The aim of the present study, therefore, was to determine the effect of ethanol contained in mouthwashes on the surface hardness of a restorative resin composite material.

\section{MATERIALS AND METHODS}

Specimens were prepared using a metallic split mold $(4 \mathrm{~mm}$ internal diameter and $6 \mathrm{~mm}$ in height) at room temperature of $24( \pm 1)^{\circ} \mathrm{C}$ and $60 \%$ relative humidity. The resin composites (Composite ${ }^{\circledR}$, Lot. QJ 26D, Prime Dental Inc, USA) were placed in the mold, covered with clear matrix strip and held between 2 glass slides in order that the specimens would be free from bubbles. From both top and bottom surfaces, the resin composites in the mold were then cured using a visible lightcuring unit $\left(\lambda=400-500 \mathrm{~nm}, f=10^{14}-10^{15} \mathrm{~Hz}\right)$ for 20 seconds with a standard distance between the light and the specimens. Taken out from the mold, the specimens were then grounded using a $\mathrm{SiC}$ paper and polished with alumina suspension. All specimens were then kept in an incubator of $37( \pm 1){ }^{\circ} \mathrm{C}$ for 24 hours to free the specimens from moisture.

Freshly taken out from the incubator, the specimens were divided into 4 groups. Three groups were tested in surface hardness after immersing the specimens in the mouthwashes and 1 group were tested without immersion. The specimens were submerged in closed-cups contain each of the commercial mouthwash and kept in an incubator $\left(37^{\circ} \mathrm{C}\right)$ for $1,2,3,7$,or 14 days. Commercial mouthwashes used in the present study were Listerine ${ }^{\circledR}$ or Bactidol $(\circledast$ which contain alcohol, or Minosepe ${ }^{\circledR}$ as the nonalchohol one, as listed in the table below.

Prior to the surface hardness testing, the specimens were carefully washed by aquadest, wiped with paper absorbent, and air dried. All specimens, including theimmersed and unimmersed

Table 1. Commercial mouthwashes used.

\begin{tabular}{ccccc}
\hline Mouthwashes & Batch No & Ethanol & Content & Manufacture \\
\hline Listerine $\circledast$ & 904 W20 & 22 VOL\% & 22 VOL\% & Pfizer, Indonesia \\
Bactidol $\circledast$ & 411072 & 9 VOL\% & 9 VOL\% & ParkeDavis, Indonesia \\
Minosepe $\circledast$ & 411072 & NA & Chlorhexidin & Minorock, Indonesia \\
\hline
\end{tabular}




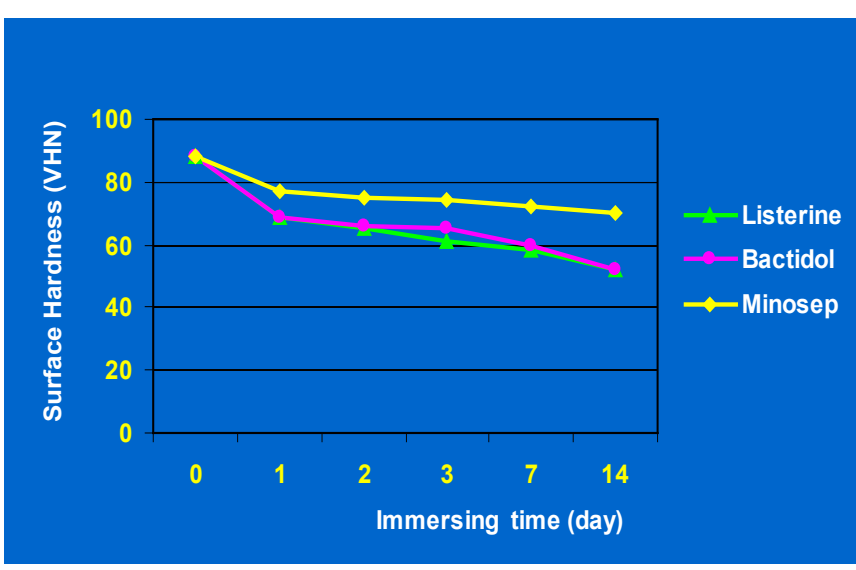

Figure 2. Changes in surface hardness of the dental resin.
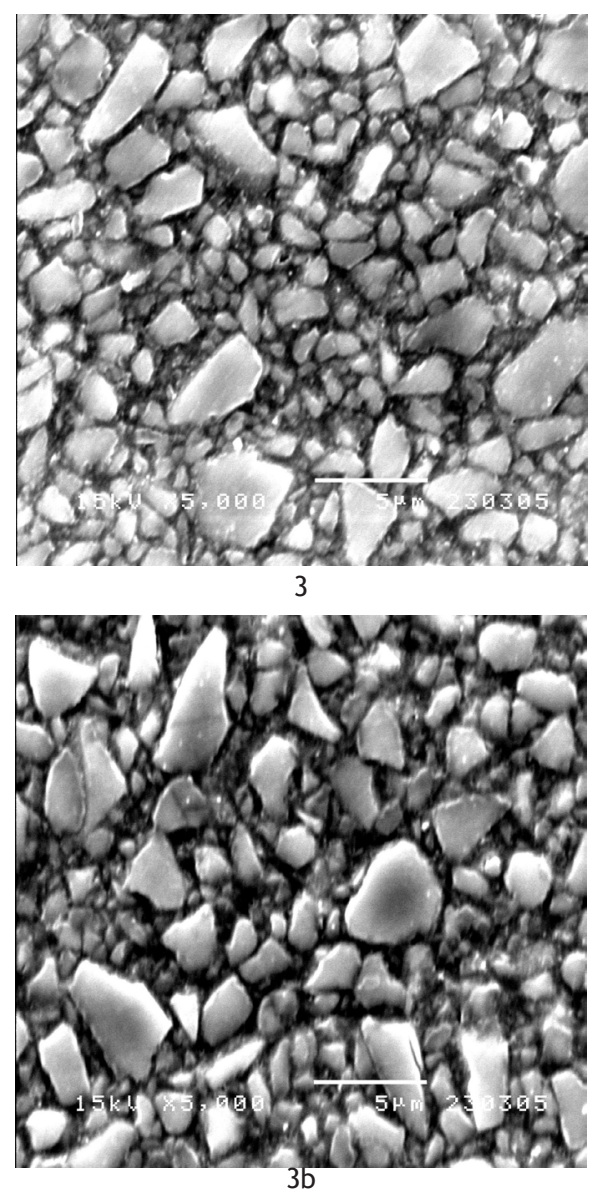

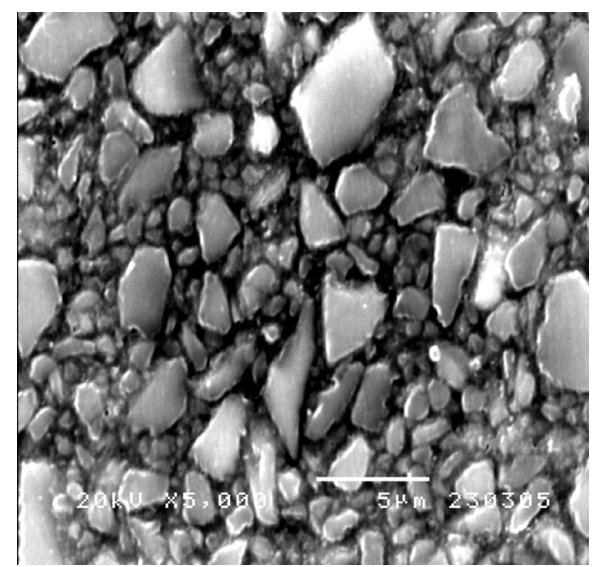

$3 a$

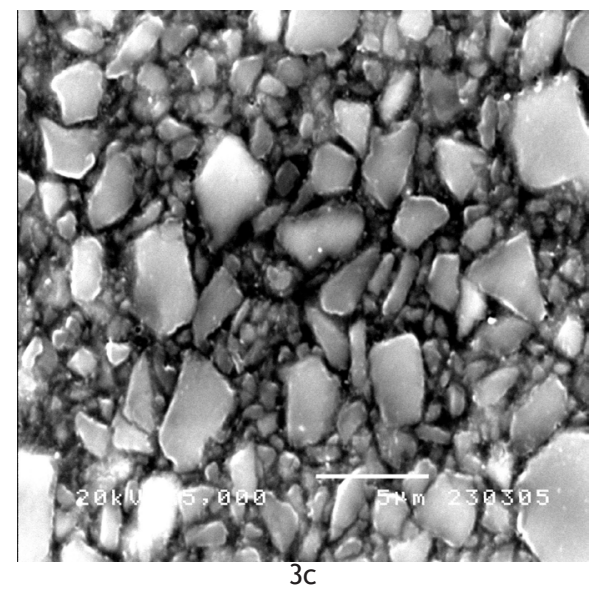

Figure 3. SEM image of dental resin composite specimens without immersing in mouthwash (3) and after immersing for 14 days in Bactidol $₫(3 a)$, Listerine $₫(3 b)$ or Minosepe $₫(3 c)$.

ones, were measured for their surface hardness testing using a $25-\mathrm{g}$ load applied through a Vickers diamond indenter over a 20-second loading cycle on a micro hardness tester (Shimadzu®, Shimadzu Corp, Japan). Five indentations were created for each specimen and were brought to a formula of the surface hardness number $(\mathrm{Hv})$ of $1854,4 \mathrm{P} / \mathrm{d}^{2}$, where $P$ was the load (gram), $d$ was the diagonal indentation length $(\mu \mathrm{m})$, and 1854,4 was the
Vickers constant. A One-way ANOVA was used to analyse the surface hardness values performed for each of the mouthwash to determine differences between the mean of $\mathrm{Hv}$ values.

\section{RESULTS}

In regard to the mouthwashes, the decrease in the surface hardness values from the composite 
specimens immersed in Bactidol ${ }^{\circledR}$ or Listerine $\AA$ were higher than those immersed in Minosepe ${ }^{\circledR}$. The composite specimens immersed for 1 day in Listerine $₫$, Bactidol $\left(\right.$, or Minosepe ${ }^{\circledR}$ showed significant $(p<0,01)$ dropped in the surface hardness values of $23 \%, 22 \%$ or $17 \%$, respectively. All specimens demonstrated a steady lowering of the values, however, they were still significant $(p<0.05)$ up to the $14^{\text {th }}$ day of immersion between each immersion times. They appeared to show a similar pattern of the surface hardness changes through the end of the immersion time.

Scanning Electronic Microscopy image showed changes on the surface texture of the resin composite specimens following immersion in the mouthwashes. With respect to the specimens immersed in mouthwashes, it appeared to be loss of small fillers from the secimens (Fig.-3a, 3b, 3c).

\section{DISCUSSION}

The alcohol mouthwashes used in the present study that contained ethanol were Bactidol $₫$ and Listerine $₫$ which were for $9 \%$ and $22 \%$, respectively, and the nonalcohol mouthwashes was Minosepe ${ }^{\circledR}$ that contained chlorhexidin. It can be assumed from the table above that water balance in Listerine $\AA$, Bactidol $₫$, or Minosepe $\AA$ were $99 \%, 90 \%$, or $77 \%$, respectively. The water molecules, i.e. $\mathrm{H}^{+}$and $\mathrm{OH}^{-}$ions elicited from water were said to be the causative factor for subsurface softening of the resin composite material. ${ }^{6-8}$ however, $\mathrm{OH}^{-}$elicited from ethanol may have been the detrimental factor to the significant drop of the surface hardness values of the resin specimens across the ageing time in this study.

In regard to the mouthwases, Bactidol ${ }^{\circledR}$ or Listerine ${ }^{\circledR}$ demonstrated higher surface hardness values than those immersed in Minosepe ${ }^{\circledR}$. This was probably because Bactidol ${ }^{\circledR}$ or Listerine $\AA$ contained more $\mathrm{OH}^{-}$ions elicited from the ethonal content when compared to Minosepe ${ }^{\circledR}$ which was a nonalcohol mouthwash. More absorbed water molecules from Bactidol ${ }^{\circledR}$ or Listerine ${ }^{\circledR}$ mouthwashes may have diffused into the polar resin matrix of the specimes and may have pushed the resin molecules apart and resulting expanded resin matrix. Exposure of water molecules into the resin composites may cause swelling of the material and would induce stress around the stiff filler Si particles of the resin composites used in the present study, and consequently, led to a degradation of the structure in the specimens surfaces, as explained below (Fig. 4):

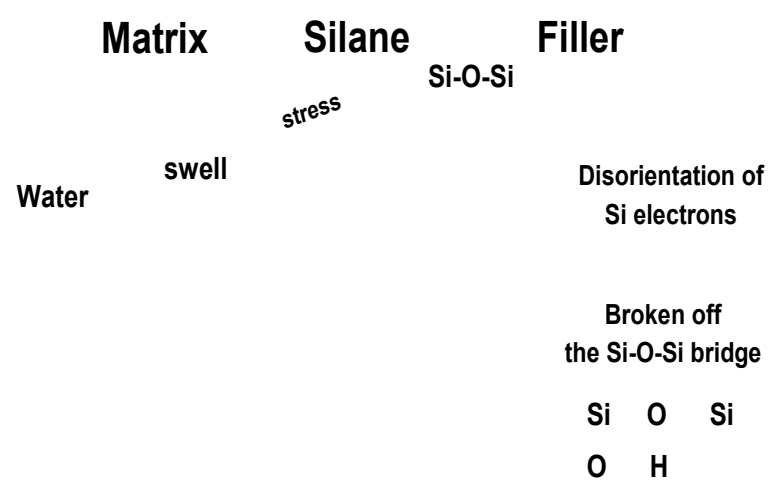

Figure 4. Degradation of the dental resin composite structure.

The swelling of the matrix further caused breakage in the Si-O-Si bridge. The oxygen atoms from water and ethanol molecules moved towards the silicon atoms resulting electron disorientation, as a result of stresses. The disorientation occured at attachments between negative poles of water molecules and the silicon nucleus, developing polar bonds. Another polar bond may developed at the other end of water molecule with its proton by bridging with the oxygen atom from the Si-O$\mathrm{Si}$ bridge. At this point, a breakage of the $\mathrm{Si}-\mathrm{O}-\mathrm{Si}$ bridge would result in a proton transfer resulting the Si-O- bridge, while the remaining $\mathrm{Si}$ - would take care of the remaining hydroxy group.

In a longer time exposure, further degradation by $\mathrm{OH}^{-}$ions may cause subsurface softening of the resin matrix. Upon loading, the load applied through the Vickers indenter on the micro hardness tester would degrade the resin composite structure with lower energy for the specimens immersed in the alcohol mouthwashes than those immersed in the nonalcohol one.

With respect to the immersing times, specimens soaked up to 14 days demonstrated significantly lower surface hardness values. Specimens in a longer time of mouthwash exposure absorbed more water molecules which meant causing more electron disorientation. Upon indentation by the Vickers indenter, more degradation would demonstrate larger indentations than those with less disoriented electrone, thus 
showing lower surface hardness values. This phenomena indicates that more penetration of water molecules into the resin matrix made the resin composites become less hard. These suggested that the hydrolysis and subsurface softening could explain degradation of the specimens immersed in mouthwashes demonstrated in the present study. As a result, individual particles dissociate from each other; thus, the SEM image showed a rough surface with voids presented and individual particles protruding as a result of loss of small fillers of the resin composites as were seen from the specimens immersed in mouthwashes (Fig. $3 a, 3 b, 3 c)$.

Compared to other research, the surface hardness values obtained at 0 time (before immersing) in the present study were higher than those revealed from Abu-bakr et al. ${ }^{11}$

Abu-bakr et al. ${ }^{11}$ presumed that different composition of the composite materials used may cause different initial hardness values. The result from the present study was not in accordance with that of Abu-Bakr et al. ${ }^{11}$ which demonstrated all specimens showed an increase in the surface hardness from 0 time to day- 1 . They proposed that this could be due to the post-curing complex maturation of the materials. The finding from the present study conformed with that from Schneider L, et al. ${ }^{6}$ which showed a decrease in the surface hardness of composites soaked in ethanol; chemical softening may took part by the $\mathrm{OH}^{-}$ions by swelling and finally softening of the matrix structure. This characteristic may also be explained by Weiner et al. ${ }^{12}$ who concluded from their finding that there were increased of specimens weight soaked in the alcoholic mouthwashes when compared to those immersed in the nonalcoholic mouthwash. The present study was also supported by Sarrett et al. ${ }^{10}$ who mentioned in their study that the effect of alcoholic beverages on composite wear depends on the alcohol composition, which should at least containing ethanol of $9 \mathrm{vol} \%$. The present study was in agreement with Sarrett et al. as alcohol composed in the mouthwashes for Bactidol $₫$ and Listerine ${ }^{\circledR}$ used in the present study were $9 \mathrm{vol} \%$ and 22 vol\%, respectively.

\section{CONCLUSION}

On immersing in mouthwashes, the dental resin composite specimens had profound implications for the surface hardness testing. Ethanol in mouthwashes initially lower the surface hardness the dental resin composite but then approached a plateau approach the second week.

\section{ACKNOWLEDGEMENTS}

We acknowledge the support from the Head of the Faculty Laboratory Centre, Faculty of Dentistry-Universitas Indonesia.

\section{REFERENCES}

1. Medeiros IS, Gomes MN, Loguercio AD, R Filho LER. Diametral tensile strength and vicker hardness of a composite after storage in different solution. J Oral Science 2007;49:616.

2. Lagouvardos PE, Pissis P, Kyritsis A, Dou Kaki D. Water sorption and water induced molecular rehability in dental composite resin. J Mater Sci 2003;14(9).

3. Ortenqren $\mathrm{U}$, Wellendorf $\mathrm{H}$, Karissson S, Ruyter IE. Water sorption and solubility of dental composites and identification of monomers released in an aqueous environment. J Oral Rehabil 2001;28(12):1106-15.

4. Indrani DJ, Cook WD, Televantos F, Tyas MJ, Harcourt JK. Fracture toughness of wateraged resin composite restorative materials. Dent Mater 1995;11:201-7.

5. Soderholm KJ, Zigan, $M$, Raga $M$, Fischlschweiger W, Bergman M. Hydrolytic degradation of dental composites. J Dent Rest 1984;63(10):1248-54.

6. Schneider L, Moraes R, Cavalcante L, Sinhoreti M, Correr-Sobrinho L, Consan S. Cross-link density evaluation through softening tests: Effect of ethanol concentration. Dent Mater 2008;24(2);199-203.

7. Ferracane JL. Hygroscopic and hydrolytic effects in dental polymer networks. J Dent Mater 2006;22:211-22.

8. Bagheri, Tyas $M$, Burrow M. Subsurface degradation of resin-based composites. Dent Mater 2003;23(18):944-51.

9. Sideridou ID, Karabela MM, Vou Vaudi EC, Papanastasiou BE. Sorption and desorption parameters of water or ethanol in light cured 
dental dimethacrylate resin. J Applied Polymer Sci 2007;107:463-75.

10. Sarret DC, Coletti DP, Peluso AR. The effects of alcoholic beverager on composite wear. Dent Mater 2000;16:62-7.

11. Abu-bakr N, Han L, Okamoto A, Iwaku M. Changes in the mechanical properties and surface texture of compomer immersed in various media. J Prosthet Dent 2000;84:44650.

12. Weiner $R$, Millstein $P$, Hoang $E$, Marshall $D$. The effect of alcoholic and non alcoholic mouthwashes on heat-treatment composite resin. Operative Dentistry 1997;R2:249-53. 